\title{
Mechanism of Huayu Jianpi Fangshi decoction in urolithiasis prevention: a randomized trial
}

\author{
Xiao-Song Gao ${ }^{1}$, Jian Pan $^{2}$, Ran Pang ${ }^{1}$, Bing Liu ${ }^{1}$, Shu-Qi Song ${ }^{1}$ \\ ${ }^{1}$ Department of Urology, China Academy of Chinese medical Sciences Guanganmen Hospital, Beijing, China; ${ }^{2}$ Department of Laboratory, China \\ Academy of Chinese medical Sciences Guanganmen Hospital, Beijing, China \\ Contributions: (I) Conception and design: XS Gao; (II) Administrative support: XS Gao; (III) Provision of study materials or patients: XS Gao, R \\ Pang, B Liu, SQ Song; (IV) Collection and assembly of data: XS Gao, J Pan, R Pang, B Liu, SQ Song; (V) Data analysis and interpretation: XS Gao, \\ J Pan, R Pang, SQ Song; (VI) Manuscript writing: All authors; (VII) Final approval of manuscript: All authors. \\ Correspondence to: Xiao-Song Gao, MD. Department of Urology, China Academy of Chinese medical Sciences Guanganmen Hospital, No. 5 of \\ Beixiange Street, Xicheng District, Beijing 100053, China. Email: gaoxiaosong56@163.com.
}

Background: This study aims to explore the mechanism of the Huayu Jianpi Fangshi decoction in urolithiasis prevention.

Methods: The present study was designed as a randomized, double-blinded, placebo-controlled clinical trial. Sixty patients with the qi stagnation and blood stasis, spleen deficiency, and dampness obstruction types of urolithiasis were randomly divided into two groups: the treatment group and the control group ( $\mathrm{n}=30$ in both groups). Patients in the treatment group were treated with the Huayu Jianpi Fangshi decoction, while patients in the control group were treated with the Huayu Fangshi placebo decoction. Both treatments were taken orally two times per day. All patients received treatment over the course of four weeks. The main outcome indicators included the Tamm-Horsfall protein (THP) expression levels, osteopontin, and inter- $\alpha$ trypsin inhibitor heavy chain 3 (ITIH3) in the patients' urine as well as changes in 24-h urinary citric acid, urinary magnesium levels, and Traditional Chinese Medicine (TCM) syndrome scores.

Results: The results of the present study revealed a significant increase in the total citric acid excretion level $(244.75 \pm 59.62$ vs. $297.48 \pm 57.91 \mathrm{mmol} / \mathrm{L}, \mathrm{P}<0.01)$, significant decrease in the total urinary THP level $(10.83 \pm 7.73$ vs. $6.37 \pm 6.10 \mathrm{mg} / \mathrm{L}, \mathrm{P}<0.05)$, significant decrease in the total $\mathrm{ITIH} 3$ level $(9.51 \pm 6.32$ vs. $6.14 \pm 4.46 \mathrm{mg} / \mathrm{L}, \mathrm{P}<0.05)$ in the patients' $24-\mathrm{h}$ urine, and a significant elevation of the total TCM syndrome score $(5 \%$ vs. $23 \%, \mathrm{P}<0.01)$ in the treatment group when compared with the control group.

Conclusions: The Huayu Jianpi Fangshi decoction can increase the excretion of urinary citric acid, decrease the expression levels of urinary THP and ITIH3 in patients with cured urolithiasis, and reduce the TCM syndrome score.

Keywords: Huayu Jianpi Fangshi decoction; stone inhibitors; urinary calculi; recurrence; randomized controlled trial

Submitted Nov 18, 2020. Accepted for publication Mar 10, 2021.

doi: 10.21037/apm-20-2295

View this article at: http://dx.doi.org/10.21037/apm-20-2295

\section{Introduction}

The urolithiasis recurrence rate is very high, and the overall recurrence rate can reach over $50 \%$ within 10 years. If the stone is removed by operation, the recurrence rate can reach up to $26 \%$ within 2 years after the operation. The recurrence rate after extracorporeal shock-wave lithotripsy in 1 year is $6.70 \%$. This rate can reach up to $41.80 \%$ in 5 years (1-3). Repeated urolithiasis increases the patient's pain and financial burden. Furthermore, extracorporeal lithotripsy and multiple operations may cause ureteral stricture, urinary tract infection, renal function damage, and other complications. In severe cases, it can lead to 
renal atrophy and renal function loss (4-6). With the improvement of China's living standards in recent years, the incidence of metabolic diseases, such as hyperglycemia and hyperlipidemia, and, combined with climate warming, the incidence of urolithiasis in China has significantly increased. Therefore, a higher and higher number of urologists are focusing their attention on determining how to effectively prevent stone occurrence. An effective method for urolithiasis recurrence prevention is the Huayu Jianpi Fangshi decoction (HJFD), which is based on Professor Liu Youfang's Huayu Niaoshi decoction in combination with Professor Gao Ronglin's academic thoughts on regulating the spleen and stomach, removing dampness, and eliminating stagnation in urolithiasis treatment. The investigators believe that the mechanism for preventing urolithiasis should improve the ion metabolism in urine, regulate the concentration and expression of the stone inhibitor, and correct patients' calculus-prone constitutions, such as spleen deficiency, blood stasis, and dampness obstruction. Understanding the etiology and pathogenesis of urolithiasis is a gradual process for traditional Chinese medicine (TCM). The Origin of Various Diseases · the Origins of Gonorrbea states that "for the patient with urolithiasis, the kidney manages the water, and the water will turn into stone, so the kidney guests sand and stone, and the kidney develops deficiency, and is invaded by heat". We can see from this that doctors have considered kidney deficiency and bladder heat crucial to urolithiasis formation for a very long period of time. Hence, the methods of promoting water, treating stranguria, and tonifying the kidney qi are often used in the treatment of calculi. Good curative effects have been achieved with these methods. Professor Youfang Liu, a famous urologist in China, proposes that qi stagnation and blood stasis are the pathogeneses of calculi retention in the urinary system. He developed the Huayu Niaoshi decoction to enrich the means of calculi treatment. However, several pieces of literature support the prevention of urolithiasis through the use of TCM. By studying with Mr. Ronglin Gao, the investigator realized that the abnormal function of the spleen and stomach may be the origin of urolithiasis formation. In the Medical True Biography, Bo Yu of the Ming Dynasty pointed out that "the taste of rich food, the things with damp and heat, or the distilled wine and fried things" are important urolithiasis occurrence factors. Furthermore, it was noted that there is a close correlation between an improper diet and urolithiasis formation. In the fingyue Whole Book, Jingyue Zhang proposes that urolithiasis is caused by the combined actions of external and internal factors. The internal cause is an improper diet, and the external cause is the steaming climate. In addition, he considered the internal cause to be key to calculi formation. Gao noted that with the warming of the climate in recent years, urolithiasis incidence is increasing. At present, people's diet structures consist mainly of high-protein and high-calorie food, leading to a yearly increase in metabolic disease incidence in China. Additionally, the age of diabetes, hypertension and hyperlipidemia onsets are constantly decreasing. These metabolic diseases are also an important cause of the increasing incidence of urolithiasis (7). This is consistent with the ancient people's idea that an improper diet is the main cause of urolithiasis. Furthermore, for thousands of years, the Chinese have mainly lived on a plant diet. Thus, their present change in diet structure can very easily lead to stomach-qi fluctuation and spleenqi insufficiency. However, the abnormal functioning of the spleen and stomach influences the transportation and transformation of water solution and the essence of water and grain. This leads to dampness originating from the interior, accumulation of heat, and damp-heat syndrome, finally resulting in the formation of urolithiasis. Gao Ronglin proposes that regulating the spleen and stomach and removing dampness and stagnation are key to preventing urolithiasis.

Therefore, this study aims to (I) verify whether the mechanism of HJFD in urolithiasis prevention will regulate the concentration and expression of the stone inhibitor and (II) explore the mechanism of TCM in preventing urolithiasis.

The following article is presented in accordance with the CONSORT reporting checklist (available at http://dx.doi. org/10.21037/apm-20-2295).

\section{Methods}

\section{Subjects}

Sixty patients with urolithiasis cured in our hospital from April 2016 to June 2017 were included in this study. The patients were randomly divided into two groups: the HJFD group and the placebo control group ( $\mathrm{n}=30$ in both groups). After 28 days of treatment, its efficacy and safety were analyzed. The Western medicine diagnosis and cure criteria of upper urinary tract calculi were based on the Chinese Urology Disease Diagnosis and Treatment Guidelines [2015] and Wu Jieping Urology [2004]. The TCM syndrome differentiation criteria of qi stagnation and blood 
stasis, spleen deficiency and dampness obstruction were formulated based on the Urology of Integrated Chinese and Western Medicine [2007]. There were three main symptoms: frequent and astringent pain during urination, turbid urine, and abdominal distention. Furthermore, there were secondary symptoms present, including stool stickiness, dry mouth, bitter mouth, and poor appetite. Additional symptoms were a dark red coloring of the tongue, a white and greasy tongue coating, and a slippery or uneven pulse. For patients with two main symptoms, one secondary symptom, and consistent tongue and pulserelated signs, the syndrome could be determined as qi stagnation and blood stasis, spleen deficiency, and dampness obstruction. The study was conducted in accordance with the Declaration of Helsinki (as revised in 2013) and approved by the Guanganmen Hospital Ethics Committee at the China Academy of Chinese Medical Sciences (No. 2016-061-KY-01). Informed consent was taken from all patients included in the study.

\section{Inclusion and exclusion criteria}

Inclusion criteria: (I) patients aged 18-60 years; (II) patients previously diagnosed with urolithiasis (upper urinary tract); (III) patients with treatment-cured urolithiasis (upper urinary tract); (IV) patients meeting the TCM criteria of qi stagnation and blood stasis, spleen deficiency, and dampness obstruction; and (V) patients who provided a signed informed consent. Exclusion criteria: (I) patients with urinary system malformation and periodical ureteral stenosis; (II) patients with urinary system tumors; (III) patients with infectious diseases of the urinary tract; (IV) patients with hyperparathyroidism; (V) patients with either serious primary diseases of the heart, brain, liver, kidney, or hematopoietic system, or serious secondary damage to the heart, brain, liver, kidney, or hematopoietic system; and (VI) patients with allergic constitutions.

\section{Treatment methods}

Treatment group: patients were treated with HJFD, at one decoction taken orally two times per day. Control group: patients were treated with the Huayu Fangshi placebo decoction, at one decoction taken orally two times per day. The course of treatment was four weeks for all patients. All included patients received the same health education regarding a daily diet for urolithiasis prevention, such as keeping the daily urine volume above $2,000 \mathrm{~mL}$. The intake of meat was recommended to be lower than $150 \mathrm{~g}$ per day. The intake of sodium salt was limited to less than $2 \mathrm{~g}$ per day. Patients were instructed not to maintain an oxalic acidrich diet. Measures were taken to prevent the test data deviation caused by the diet and water consumption.

\section{Main observation indicators}

Main outcome indicators: (I) the protein expression levels of the Tamm-Horsfall protein (THP), osteopontin (OPN), and inter- $\alpha$-trypsin inhibitor heavy chain 3 (ITIH 3 ) in urine; (II) the 24-h urinary citric acid and magnesium levels; and (III) the TCM syndrome score. Secondary outcome indicators: (I) urine $\mathrm{pH}$ value; (II) 24-h urinary oxalic acid, urinary acid, urinary calcium, and urinary phosphorus levels; and (III) blood uric acid, magnesium, phosphorus, and calcium levels. All indicators were detected at the time of enrollment and at four weeks after the medication.

\section{Curative effect evaluation criteria}

The index changes in these two groups were either compared before and after treatment, or between the two groups. Main outcome indicators were (I) the THP, OPN and ITIH3 protein-expression levels in the patients' urine (both an intra-group comparison before and after treatment and an inter-group comparison were conducted according to the measurement data); (II) 24-h urinary citric acid and magnesium levels: the measurement data before and after treatment and between the groups were compared, respectively; (III) curative effect criteria for the TCM syndrome (cure: the total score decreased by $\geq 90 \%$ compared with the score obtained before treatment; excellent: the total score decreased by $70-90 \%$ compared with the score obtained before treatment; effective: the total score decreased by 30-70\% compared with the score obtained before treatment; ineffective: the total score decreased by $<30 \%$ compared with the score obtained before treatment). TCM syndrome raters were blinded. Secondary outcome indicators were (I) urine $\mathrm{pH}$ value: the measurement data were compared before and after treatment and between groups; (II) 24-h urinary oxalic acid, urinary uric acid, urinary calcium, and urinary phosphorus levels: the measurement data were compared before and after treatment and between groups; (III) serum uric acid, magnesium, phosphorus, and calcium levels: the measurement data were compared before and after treatment and between groups. 
Table 1 Comparison of THP, OPN and ITIH3 (mg/L)

\begin{tabular}{lcccc}
\hline Group & Treatment period & THP & OPN & ITIH3 \\
\hline Treatment group & Before treatment & $12.48 \pm 10.23$ & $7.89 \pm 6.43$ & $10.18 \pm 7.81$ \\
& After treatment & $6.37 \pm 6.10^{\star}$ & $7.48 \pm 5.24$ & $6.14 \pm 4.46^{*}$ \\
Control group & Before treatment & $11.57 \pm 9.14$ & $8.33 \pm 6.18$ & $9.86 \pm 6.63$ \\
& After treatment & $10.83 \pm 7.73$ & $8.22 \pm 6.38$ & $9.51 \pm 6.32$ \\
\hline
\end{tabular}

Compared with the control group, ${ }^{*} \mathrm{P}<0.05$. THP, Tamm-Horsfall protein; OPN, osteopontin; ITIH3, inter- $\alpha$-trypsin inhibitor heavy chain 3.

\section{Statistical analysis}

In the present study, data were analyzed using the statistical software SPSS 17.0. Continuous data were expressed using the mean \pm standard deviation, and count data were expressed as a percentage (\%). Normality was tested using the Shapiro-Wilk test, homogeneity of variance was determined using the F-test, and the inter-group comparison was conducted using the $t$-test. Both the non-normally distributed means of multiple samples and the normally distributed means of multiple samples with a heterogeneity of variance were compared using nonparametric tests. Count data were compared using the Chi-square test. A $\mathrm{P}$ value of $<0.05$ (two-sided) was considered statistically significant. The dataset was a PP with no missing value.

\section{Results}

\section{General data}

A total of 60 patients were included in the present study. These patients were randomly divided into two groups: the treatment group and the control group ( $\mathrm{n}=30$ in both groups). After 28 days of treatment, its curative effect and safety were analyzed. Finally, 57 patients completed the clinical trial in accordance with the requirements of the scheme. Of these patients, 29 were assigned to the treatment group and 28 were assigned to the control group. 45 of the patients were male and 12 were female. All patients were aged 19-60 years, the average age being 42 years. The patients' disease courses were ranged 6-240 months, with an average disease course of 32 months. The differences between these two groups in age, gender ratio, course of disease, and the TCM syndrome score were not statistically significant $(\mathrm{P}>0.05)$. Hence, the two groups were comparable. The differences in THP, OPN and ITIH3 between the two groups before treatment were not statistically significant $(\mathrm{P}>0.05)$. Hence, the two groups were comparable. The differences in urinary $\mathrm{pH}$ value, 24-h urinary citric acid, urinary magnesium, urinary phosphorus, urinary calcium, urinary oxalic acid, urinary uric acid, blood phosphorus, blood magnesium, blood calcium, and blood uric acid levels between the two groups before treatment were not statistically significant $(\mathrm{P}>0.05)$. Hence, these two groups were comparable.

\section{Comparison of THP, OPN, and ITIH3}

The difference in urinary THP concentration in the treatment group before and after treatment was highly statistically significant $(\mathrm{P}<0.01)$. The difference in urinary THP concentration between the treatment group and control group after treatment was also statistically significant $(\mathrm{P}<0.05)$. The THP concentration after treatment was lower than before treatment. The difference in urinary OPN concentration in the treatment group before and after treatment was not statistically significant $(\mathrm{P}>0.05)$. The difference in urinary OPN concentration between the treatment group and control group after treatment was not statistically significant $(\mathrm{P}>0.05)$. The difference between the ITIH 3 concentration in the treatment group before and after treatment was highly statistically significant $(\mathrm{P}<0.01)$. The difference in ITIH3 concentration between the treatment group and control group after treatment was not statistically significant $(\mathrm{P}>0.05)$. The ITIH3 concentration after treatment was lower than before treatment (Table 1).

\section{Comparison of 24-b urinary citric acid and magnesium}

The difference in 24-h urinary citric acid levels in the treatment group before and after treatment was statistically significant $(\mathrm{P}<0.01)$. The difference in 24-hour urinary citric acid between the treatment group and control group after treatment was highly statistically significant $(\mathrm{P}<0.01)$. The 24-h urinary citric acid levels after treatment were significantly higher than before treatment. The difference 
Table 2 Comparison of 24-hour urinary citric acid and urinary magnesium

\begin{tabular}{lccc}
\hline Group & $\begin{array}{c}\text { Treatment } \\
\text { period }\end{array}$ & $\begin{array}{c}\text { Urinary citric acid } \\
(\mathrm{mg} / \mathrm{L})\end{array}$ & $\begin{array}{c}\text { Urinary magnesium } \\
(\mathrm{mmol} / \mathrm{L})\end{array}$ \\
\hline $\begin{array}{l}\text { Treatment } \\
\text { group }\end{array}$ & $\begin{array}{c}\text { Before } \\
\text { treatment } \\
\text { After treatment }\end{array}$ & $241.03 \pm 62.43$ & $2.52 \pm 0.314$ \\
& $297.48 \pm 57.91^{\#}$ & $2.49 \pm 0.30$ \\
$\begin{array}{l}\text { Control } \\
\text { group }\end{array}$ & $\begin{array}{c}\text { Before } \\
\text { treatment }\end{array}$ & $240.79 \pm 62.77$ & $2.52 \pm 0.313$ \\
& After treatment & $244.75 \pm 59.62$ & $2.52 \pm 0.29$ \\
\hline
\end{tabular}

Compared with the control group, ${ }^{\#} \mathrm{P}<0.01$.

Table 3 Comparison of the curative effect of TCM syndromes

\begin{tabular}{lcc}
\hline Curative effect & $\begin{array}{c}\text { Treatment } \\
\text { group, } \mathrm{n}(\%)\end{array}$ & $\begin{array}{c}\text { Control } \\
\text { group, } \mathrm{n}(\%)\end{array}$ \\
\hline Recovery & $5(17.2)$ & $1(3.6)$ \\
Markedly effective & $10(34.5)$ & $3(10.7)$ \\
Effective & $8(27.6)$ & $4(14.3)$ \\
No effect & $6(20.7)$ & $20(71.4)$ \\
Total effective rate & $23(79.3)^{\#}$ & $8(28.6)$ \\
\hline
\end{tabular}

Compared with the control group, ${ }^{\#} \mathrm{P}<0.01$. TCM, traditional Chinese medicine.

in the 24-h urinary magnesium levels in the treatment group before and after treatment was not statistically significant $(\mathrm{P}>0.05)$. The difference in the 24-h urinary magnesium levels between the treatment group and control group after treatment was not statistically significant $(\mathrm{P}>0.05)$. These results suggest that there was no change in the total magnesium excretion in 24-h urine before and after treatment (Table 2).

\section{Comparison of the curative effect of TCM syndromes}

Regarding the curative effects of TCM syndromes in the two groups after treatment, the total effective rate was $79.3 \%$ in the treatment group and $28.6 \%$ in the control group. The difference between these two groups was statistically significant $(\mathrm{P}<0.01)$. This result suggests that HJFD can effectively improve the clinical symptoms of patients with urinary system diseases, change the calculus-prone constitution of patients with blood stasis, spleen deficiency, and dampness obstruction, and play a fundamental role in urolithiasis recurrence prevention (Table 3).
Comparison of urinary $p H$ value, 24-b urinary oxalic acid, urinary uric acid, urinary calcium, and urinary phosphorus levels

The urinary $\mathrm{pH}$ value in the treatment group was higher after treatment than before treatment. The difference was highly statistically significant $(\mathrm{P}<0.01)$. The differences in urinary $\mathrm{pH}$ values between the treatment group and control group after treatment were also statistically significant $(\mathrm{P}<0.01)$. The urinary $\mathrm{pH}$ value was higher in the treatment group than in the control group. The urinary calcium level was lower in the treatment group after treatment than before treatment. The difference was highly statistically significant $(\mathrm{P}<0.01)$. The differences in urinary calcium between the treatment group and control group after treatment were highly statistically significant $(\mathrm{P}<0.01)$. The urinary calcium level showed a larger decrease in the treatment group than in the control group. The differences in the 24-h urinary oxalic acid, urinary uric acid, urinary calcium, and urinary phosphorus levels in the treatment group before and after treatment were not statistically significant $(\mathrm{P}>0.05)$. The differences in the 24-h urinary oxalic acid, urinary uric acid, urinary calcium, and urinary phosphorus levels between the treatment group and control group after treatment were not statistically significant $(\mathrm{P}>0.05$, Table 4).

\section{Comparison of serum uric acid, magnesium, phosphorus, and calcium levels}

The differences in serum uric acid, magnesium, phosphorus, and calcium levels in the treatment group before and after treatment were not statistically significant $(\mathrm{P}>0.05)$. The differences in serum uric acid, magnesium, phosphorus, and calcium levels between the treatment group and control group after treatment were not statistically significant $(\mathrm{P}>0.05$, Table 5).

\section{Discussion}

The results of this study revealed that after four weeks of treatment, the total excretion level of citric acid in 24-h urine was significantly higher in the treatment group than in the control group and that the difference in the total excretion level of magnesium in 24-h urine between the two groups was not statistically significant. In the treatment group, the urinary THP concentration was lower after 4 weeks of treatment than before treatment. The difference in the 
Table 4 Comparison of urinary $\mathrm{pH}$ value, 24-hour urinary oxalic acid, urinary uric acid, urinary calcium and urinary phosphorus

\begin{tabular}{lccccc}
\hline Group & $\begin{array}{c}\text { Treatment } \\
\text { period }\end{array}$ & $\begin{array}{c}\text { Urinary } \\
\mathrm{pH} \text { value }\end{array}$ & $\begin{array}{c}\text { 24-hour urinary } \\
\text { oxalic acid }\end{array}$ & $\begin{array}{c}\text { Urinary } \\
\text { uric acid }\end{array}$ & $\begin{array}{c}\text { Urinary } \\
\text { calcium }\end{array}$ \\
\hline $\begin{array}{l}\text { Treatment } \\
\text { group }\end{array}$ & Before treatment & $6.19 \pm 0.53$ & $0.58 \pm 0.068$ & $4.52 \pm 0.64$ & $7.51 \pm 0.73$ \\
phosphorus
\end{tabular}

Compared with the control group, $\stackrel{\#}{\mathrm{P}}<0.01$.

Table 5 Comparison of serum uric acid, serum magnesium, serum phosphorus, serum calcium

\begin{tabular}{lccccc}
\hline Group & Treatment period & Serum uric acid $(\mu \mathrm{mmol} / \mathrm{L})$ & Serum magnesium $(\mathrm{mmol} / \mathrm{L})$ & Serum phosphorus & Serum calcium \\
\hline Treatment & Before treatment & $319.07 \pm 53.92$ & $0.88 \pm 0.54$ & $1.316 \pm 0.17$ & $2.51 \pm 0.19$ \\
group & After treatment & $318.39 \pm 53.95$ & $0.885 \pm 0.053$ & $1.313 \pm 0.17$ & $2.49 \pm 0.15$ \\
& & $318.17 \pm 54.44$ & $0.88 \pm 0.04$ & $1.32 \pm 0.171$ & $2.50 \pm 0.18$ \\
Control & Before treatment & $318.46 \pm 54.21$ & $0.873 \pm 0.040$ & $1.315 \pm 0.15$ & $2.48 \pm 0.14$ \\
group & After treatment & &
\end{tabular}

urinary THP reduction rates was statistically significant when compared with the control group. The difference in urinary OPN concentration in the treatment group before and after treatment was not statistically significant. The difference in urinary OPN concentration between the treatment group and control group after treatment was not statistically significant. The difference in the urinary ITIH3 concentration in the treatment group before and after treatment was statistically significant. The difference in urinary ITIH3 concentration between the treatment group and control group after treatment was statistically significant. The urinary ITIH3 concentration significantly decreased after treatment. In the treatment group, HJFD could significantly reduce the TCM syndrome score after four weeks of treatment. The total effectiveness rate in the treatment group was $79.3 \%$, while the total effectiveness rate in the control group was $28.6 \%$. The difference was statistically significant.

The cause of urolithiasis is complex, on account of the combined actions of many different factors (8-10). These pathogenic factors can be divided into two groups: individual factors and environmental factors. Individual factors include local factors, metabolic abnormalities, and genetic factors. Environmental factors include the climate, diet, and occupation. However, in over $50 \%$ of patients with urolithiasis, a clear cause of the condition cannot be found, leading to many difficulties regarding urolithiasis prevention. Furthermore, many patients with urolithiasis have paid great attention to diet and water consumption, but their calculi recurrence remains high. Citric acid drugs have a definite effect on calculi prevention (11) but need to be used over a long period of time. They can also cause several side effects, such as electrolyte disorder and gastrointestinal tract stimulation. The Chinese have gained a large amount of experience in the treatment of urolithiasis with herbal medicine, which has a clear effect on urolithiasis prevention and fewer side effects. In addition, it can improve the calculus-prone constitution of patients with overall adjustment. However, there is a lack of systematic research verifying this (12).

This affected the designing of the HJFD used in the present study, which was based on the Huayu Niaoshi decoction from our department in combination with Gao's academic thoughts on urolithiasis prevention and treatment through regulating the spleen and stomach and eliminating dampness and stagnation. The drugs for bloodbreaking and promoting the circulation of qi, such as Saponifia and Common Burreed Rhizome, were removed, and the membranes of chicken gizzards and Atractylodes macrocephala, which have the effects of tonifying the spleen and removing dampness and food retention, were added instead. This decoction is composed of Rbizoma curcumae, Atractylodes macrocephala, Poria cocos, membranes of chicken gizzards, peach seed, dried tangerine or orange peel, Lysimachia christinae, stony reed, and oriental waterplantain rhizome, which can prevent the occurrence of urolithiasis 
by activating blood circulation, removing blood stasis, tonifying the spleen, and removing dampness. This has had a significant effect on urolithiasis recurrence prevention in the 5 years of the decoction's application.

It was found in a study on the formation of urolithiasis that there are several components in the human body that can inhibit the nucleation, aggregation, and growth of crystals in urine. These components, called calculus inhibitors, play a very important role in inhibiting the formation of urolithiasis. Calculus inhibitors are divided into macromolecular calculus inhibitors and micromolecular calculus inhibitors. Micromolecular calculus inhibitors include citric acid, urinary magnesium, and pyrophosphate. Macromolecular calculus inhibitors are mainly proteins, and include THP, OPN, ITIH3, and urinary prothrombin fragment 1 (13-15). In normal human urine, calculus inhibitors are highly active. Hence, calculi are not formed easily. However, when the concentration or activity of calculus inhibitors is decreased or changed due to various reasons, the inhibition of calculi formation can be reduced, causing urolithiasis to form easily (16-18). The investigators in this study believe that the mechanism of HJFD for urolithiasis formation prevention may be correlated with the regulation of the content and active expression of urinary calculus inhibitors. The abovementioned clinical trials revealed that HJFD can increase the excretion of urinary citric acid, thereby alkalizing urine and preventing the nucleation and aggregation of calculus crystals. These can, in turn, downregulate the abnormal expression of THP and ITIH3, reduce the excretion of calcium, and improve the $\mathrm{pH}$ value in the urine of patients with calculus. All of these have a positive significance in the prevention of urolithiasis. Furthermore, HJFD can improve the clinical symptoms of qi stagnation and blood stasis, spleen deficiency, and dampness obstruction in patients with urolithiasis, effectively reduce the TCM syndrome score, and correct the calculus-prone constitution of spleen deficiency, blood stasis, and the dampness obstruction of patients with urolithiasis. Overall, HJFD can regulate the content and active expressions of the main urinary calculus inhibitors and regulate the patients' metabolisms. These results suggest that this decoction is a promising research prospect in the prevention of urinary calculus recurrence.

The present study still has several limitations. First, it is a single-center clinical trial with a small sample size. Hence, multi-center clinical trials with larger sample sizes are still necessary. Second, the investigation of the mechanism in the present study was not conducted deeply enough. Hence, further research from the perspective of molecular biology is necessary.

\section{Conclusions}

HJFD can increase the excretion levels of micromolecular calculus inhibitor urinary citric acid, downregulate the abnormal expressions of urinary THP and urinary ITIH3, improve the main clinical symptoms of qi stagnation and blood stasis, spleen deficiency, and dampness obstruction, effectively reduce the TCM syndrome score, and correct the calculus-prone constitution of patients with blood stasis, spleen deficiency, and dampness obstruction.

\section{Acknowledgments}

Funding: None.

\section{Footnote}

Reporting Checklist: The authors have completed the CONSORT reporting checklist. Available at http://dx.doi. org/10.21037/apm-20-2295

Data Sharing Statement: Available at http://dx.doi. org/10.21037/apm-20-2295

Conflicts of Interest: All authors have completed the ICMJE uniform disclosure form (available at http://dx.doi. org/10.21037/apm-20-2295). The authors have no conflicts of interest to declare.

Etbical Statement: The authors are accountable for all aspects of the work in ensuring that questions related to the accuracy or integrity of any part of the work are appropriately investigated and resolved. The study was conducted in accordance with the Declaration of Helsinki (as revised in 2013) and approved by the Guanganmen Hospital Ethics Committee at the China Academy of Chinese Medical Sciences (No. 2016-061-KY-01). Informed consent was taken from all patients included in the study.

Open Access Statement: This is an Open Access article distributed in accordance with the Creative Commons Attribution-NonCommercial-NoDerivs 4.0 International License (CC BY-NC-ND 4.0), which permits the noncommercial replication and distribution of the article with the strict proviso that no changes or edits are made and the 
original work is properly cited (including links to both the formal publication through the relevant DOI and the license). See: https://creativecommons.org/licenses/by-nc-nd/4.0/.

\section{References}

1. Seitz C, Bach T, Bader M, et al. Update of the 2Sk guidelines on the diagnostics, treatment and metaphylaxis of urolithiasis (AWMF register number 043-025): What is new? Urologe A 2019;58:1304-12.

2. Astroza GM, Sarras M, Salvado JA, et al. Early ureteroscopic treatment in patients with urosepsis associated with ureteral calculi is a safe approach. A pilot study. Cent European J Urol 2019;72:163-8.

3. Hunprasit V, Schreiner PJ, Bender JB, et al. Epidemiologic evaluation of calcium oxalate urolithiasis in dogs in the United States: 2010-2015. J Vet Intern Med 2019;33:2090-5.

4. Wang B, Ye JF, Zhao L, et al. Multiple endoscopy for the treatment of upper urinary tract calculi following ileal conduit: a case report and literature review. Beijing Da Xue Xue Bao Yi Xue Ban 2017;49:733-5.

5. Shi B, Hong X, Yu J. Management of unilateral staghorn renal stones with concurrent urinary tract infections by retroperitoneal laparoscopic pyelolithotomy with prolonged renal posterior lower segment incision. Exp Ther Med 2019;18:366-72.

6. Alaoui AE, Boté HE, Ziouani O, et al. The role of open nephrolithotomy in the treatment of coralliform stones: a series of 53 patients. Pan Afr Med J 2019;32:110.

7. Haymann JP. Metabolic disorders: stones as first clinical manifestation of significant diseases. World J Urol 2015;33:187-92.

8. Alatab S, Pourmand G, El Howairis Mel F, et al. National Profiles of Urinary Calculi: a Comparison Between

Cite this article as: Gao XS, Pan J, Pang R, Liu B, Song SQ. Mechanism of Huayu Jianpi Fangshi decoction in urolithiasis prevention: a randomized trial. Ann Palliat Med 2021;10(4):43204327. doi: 10.21037/apm-20-2295
Developing and Developed Worlds. Iran J Kidney Dis 2016;10:51-61.

9. Marangella M. Medical management of urinary calculi: up to date 2016. Urologia 2016;83:110-23.

10. Chigerwe M, Shiraki R, Olstad EC, et al. Mineral composition of urinary calculi from potbellied pigs with urolithiasis: 50 cases (1982-2012). J Am Vet Med Assoc 2013;243:389-93.

11. Skolarikos A, Straub M, Knoll T, et al. Metabolic evaluation and recurrence prevention for urinary stone patients: EAU guidelines. Eur Urol 2015;67:750-63.

12. Li H, Deng S, Wang J, et al. Traditional Chinese medicine on treating ureteral calculi: A systematic review and metaanalysis protocol. Medicine (Baltimore) 2019;98:e17057.

13. Khan A. Prevalence, pathophysiological mechanisms and factors affecting urolithiasis. Int Urol Nephrol 2018;50:799-806.

14. Ruiz-Agudo E, Burgos-Cara A, Ruiz-Agudo C, et al. A non-classical view on calcium oxalate precipitation and the role of citrate. Nat Commun 2017;8:768.

15. Mulay SR, Eberhard JN, Desai J, et al. Hyperoxaluria Requires TNF Receptors to Initiate Crystal Adhesion and Kidney Stone Disease. J Am Soc Nephrol 2017;28:761-8.

16. Khan SR, Joshi S, Wang W, et al. Regulation of macromolecular modulators of urinary stone formation by reactive oxygen species: transcriptional study in an animal model of hyperoxaluria. Am J Physiol Renal Physiol 2014;306: F1285-95.

17. Doizi S, Rodgers K, Poindexter J, et al. Assessment of Urinary Inhibitor or Promoter Activity in Uric Acid Nephrolithiasis. J Urol 2016;195:1799-804.

18. Manfredini R, De Giorgi A, Storari A, et al. Pears and renal stones: possible weapon for prevention? A comprehensive narrative review. Eur Rev Med Pharmacol Sci 2016;20:414-25. 